University of Wollongong

Research Online

Faculty of Engineering and Information

Faculty of Engineering and Information

Sciences - Papers: Part B

Sciences

2017

Evaluating the effectiveness of a machine learning approach based on response time and reliability for islanding detection of distributed generation

Mollah R. Alam

American International University-Bangladesh, mra497@uowmail.edu.au

Kashem M. Muttaqi

University of Wollongong, kashem@uow.edu.au

Abdesselam Bouzerdoum

University of Wollongong, bouzer@uow.edu.au

Follow this and additional works at: https://ro.uow.edu.au/eispapers1

Part of the Engineering Commons, and the Science and Technology Studies Commons

Research Online is the open access institutional repository for the University of Wollongong. For further information contact the UOW Library: research-pubs@uow.edu.au 


\title{
Evaluating the effectiveness of a machine learning approach based on response time and reliability for islanding detection of distributed generation
}

\author{
Abstract \\ Conventional relays, such as vector surge relay, frequency relay and rate-of-change-of-frequency relay, are \\ usually employed for islanding detection; however, these conventional relays fail to detect islanding \\ incidents in the presence of small power imbalance inside the islanded system. This study presents an \\ islanding detection approach for synchronous type distributed generation using multiple features \\ extracted from network variables and a support vector machine (SVM) classifier. Features are extracted \\ from a sliding temporal window, whose width is selected so as to achieve the highest detection rate at a \\ fixed false alarm rate. The SVM classifier is trained with linear, polynomial and Gaussian radial basis \\ function kernels, and the parameters of the kernels are tuned to improve the classification performance. \\ The application of the proposed method is illustrated for islanding cases associated with different power \\ imbalance conditions, including small power imbalance conditions associated with the non-detection \\ zone of conventional relays. Furthermore, variation of detection time as a function of power imbalance \\ scenarios, which involve all probable combinations of deficit of active/reactive and excess of active/ \\ reactive power imbalance, is assessed in the testing phase. The performance of the proposed approach \\ is evaluated and compared with those of conventional relays in terms of reliability and response time of \\ islanding detection.

\section{Disciplines} \\ Engineering | Science and Technology Studies

\section{Publication Details} \\ M. Rezaul. Alam, K. M. Muttaqi \& A. Bouzerdoum, "Evaluating the effectiveness of a machine learning \\ approach based on response time and reliability for islanding detection of distributed generation," IET \\ Renewable Power Generation, vol. 11, (11) pp. 1392-1400, 2017.
}




\title{
Evaluating the Effectiveness of a Machine Learning Approach Based on Response Time and Reliability for Islanding Detection of Distributed Generation
}

\author{
M. R. Alam ${ }^{1 *}$ K. M. Muttaqi², A. Bouzerdoum ${ }^{2,3}$ \\ ${ }^{1}$ Department of Electrical \& Electronic Engineering, American International University- \\ Bangladesh (AIUB), Banani, Dhaka 1212, Bangladesh \\ ${ }^{2}$ School of Electrical, Computer and Telecommunications Engineering, University of \\ Wollongong, NSW 2500, Australia \\ ${ }^{3}$ College of Science and Engineering, Hamad Bin Khalifa University (HBKU), Education \\ City, Doha, Qatar. \\ *mra015@aiub.edu
}

\begin{abstract}
Conventional relays, such as vector surge relay (VSR), frequency relay (FR) and ROCOF (rateof-change-of-frequency) relay, are usually employed for islanding detection; however, these conventional relays fail to detect islanding incidents in the presence of small power imbalance inside the islanded system. This article presents an islanding detection approach for synchronous type DG using multiple features extracted from network variables and a support vector machine (SVM) classifier. Features are extracted from a sliding temporal window, whose width is selected so as to achieve the highest Detection Rate (DR) at a fixed False Alarm (FA) rate. The SVM classifier is trained with linear, polynomial and Gaussian RBF kernels, and the parameters of the kernels are tuned to improve the classification performance. The application of the proposed method is illustrated for islanding cases associated with different power imbalance conditions, including small power imbalance conditions associated with the non-detection zone (NDZ) of conventional relays. Furthermore, variation of detection time as a function of power imbalance scenarios, which involve all probable combinations of deficit of active/reactive and excess of active/reactive power imbalance, is assessed in the testing phase. The performance of the proposed approach is evaluated and compared with those of conventional relays in terms of reliability and response time of islanding detection.
\end{abstract}

Key-words: Distributed generation, frequency relay, islanding detection, non-detection zone, ROCOF relay, sliding window, support vector machine, and vector surge relay.

\section{Introduction}

The penetration of distributed generation (DG) is forcing the electricity system planners and operators to develop standards, referred to as Interconnection Guidelines (IG) of distributed resources with electric power system (EPS) [1]-[2]. An essential requirement for IG is the protection of DG islanding, also known as the protection of "loss of mains". Islanding is a situation when a portion of EPS is energized solely by one or more local DG systems while that portion of the EPS is electrically isolated from the rest of the EPS [1]. This electrical isolation may occur due to switching of feeder, operation of switchgear and/or action of fault clearing, etc. According to IG [1]-[2], islanded DG must be disconnected quickly in order to avoid possible hazardous conditions, such as power quality degradation and damage of utility and customer equipment. The IEEE 1547-2003 standard recommends that the DG disconnection time should 
be less than 2 seconds. However, recent trend of fast automatic reclosing may have adverse effect (e.g. causing serious damage)on the islanded synchronous DGs as well as on neighbouring utility equipment if 2 seconds of DG disconnection time-range is practiced [3]. Therefore, this disconnection time-range may need to be reduced to permit the disconnection of islanded DG prior to completing the first reclosing operation [1]-[2], [4].

Normally, islanding detection is performed by a special protection device, namely islanding detection relay whose operating principles are based on active, passive, hybrid and remote communication methods [5]. Active methods are reliable, but they incur a power quality issue as periodic disturbance is introduced in this method. Remote communication based methods, such as power line signaling and transfer trip, are the most reliable scheme, but they require an enormous cost on infrastructure development. Passive methods have low cost but display poor performance when power imbalance in the islanded network is very small [5]-[8]. The conventional relays, for instance, frequency relay (FR), vector surge relay (VSR), and ROCOF (rate-of-change-of-frequency) relays, are operated on the principle of passive islanding detection methods. ROCOF and VSR relays have a strong correlation with active power imbalance of the islanded system. The performance of VSR and ROCOF relays deteriorates when the power imbalance drops below a specified threshold, which gives rise to the limitation of Non-Detection Zone (NDZ) [9]. Hybrid detection scheme is a combination of active/passive methods; it aims to maximize the advantages and minimizes the disadvantages of both methods.

Considering cost and reliability, machine learning approach has been shown recently as a potential tool for islanding detection [3], [5], [10]-[13]. To this end, this article proposes and assesses the effectiveness of a passive islanding detection method which involves multiple features and a support vector machine (SVM) classifier. In [5] and [12], an SVM based approach wasproposed to demonstrate the reliability of the method in terms of accuracy of islanding detection with a minor risk of nuisance tripping (false alarm). The classification performance was assessed based on featuresextracted from a window spanning 10 cycles in time [5], [12]. In [12], the SVM based approach was compared with a decision tree based approach presented in [13]. The same test network and test cases were considered for the comparative study. Test results indicate that the SVM based technique can detect all islanding events in the test cases, unlike the method presented in [13], which fails to detect three islanding cases in the presence of 5\% power imbalance as reported by the authors. However, for real-time applications the SVM based relay (SVMR) needs to be investigated for its detection accuracy and speed, i.e., response-time or detection-time. Therefore, this study incorporates a sliding time window, and investigates the performance of the SVMR as a function of the window length. The window size imposes a tradeoff between the speed 
and detection accuracy: increasing the width of the window increases the detection accuracy at the expense of response time. In order to achieve an optimum tradeoff, the smallest window size is selected that achieves the highest detection rate at a given false alarm rate. The SVMR is assessed considering the islanding cases with all possible combinations of deficit and excess of active/reactive power imbalance. Moreover, a majority voting rule is proposed to assess the effectiveness of SVMR under real-time environment considering response-time and accuracy.

The remainder of the paper is structured as follows. Section 2 presents the proposed methodology describing the feature extraction, theory of support vector machine, training and testing of SVM for the detection of islanding. Section 3 investigates the evaluation of the method using the features obtained from different window sizes. In this section, the islanding cases involving all possible combinations of power imbalance scenarios are also tested. In section 4, the proposed approach is examined in various realistic scenarios that may be encountered under real-time operation. Comparative analysis with conventional relays, in terms of reliability and detection time, is also carried out in this section. Section 5 concludes the paper.

\section{A Machine Learning Approach for Islanding Detection}

The proposed methodology is narrated in three sub-sections. Section 2.1 describes the process of feature extraction from plausible islanding and non-islanding events. The theory of support vector machine (SVM), relevant to the classification of two classes, is briefly discussed in section 2.2. Finally, section 2.3 narrates the training and testing procedure of SVM.

\subsection{Feature Extraction}

A3-bus radial distribution network containing distribution line, upstream grid and a synchronous generator (SG) type DG (see Fig. 1) is considered for demonstrating the process of feature extraction.

The behavior of network variables can be examined from the vector diagram shown in Fig. 2. The diagram (i.e. Fig. 2) shows the voltage behavior at DG connection point; it is drawn based on the values obtained by simulating the islanding scenarios generated by opening the circuit breaker (CB) in the system shown in Fig. 1. During islanding period, the system is composed by the synchronous generator and load only. At this instant, the synchronous generator starts to feed a smaller (or larger) load because the current injected into (or provided by) the utility side is suddenly interrupted. Thus, the generator begins to accelerate (or decelerate) its rotor speed to reduce the gap of power mismatch. Therefore, the terminal voltage and angle with respect to a reference are affected; which are illustrated in one pre-islanding (solid line) and two post-islanding scenarios (dashed and dash-dotted lines) of Fig. 2. The dashed and dash-dotted lines 
represent the behavior of voltage at the connection point of DG during $6^{\text {th }}$ and $7^{\text {th }}$ cycle after the onset of islanding. In islanded mode, change of voltage behavior in each cycle is observed, which is influenced by the dynamics of synchronous generator. Note that voltage and current phasors of Fig.2 is extracted at each cycle by applying DFT (Discrete Fourier Transform) on the instantaneous voltage and current signals.

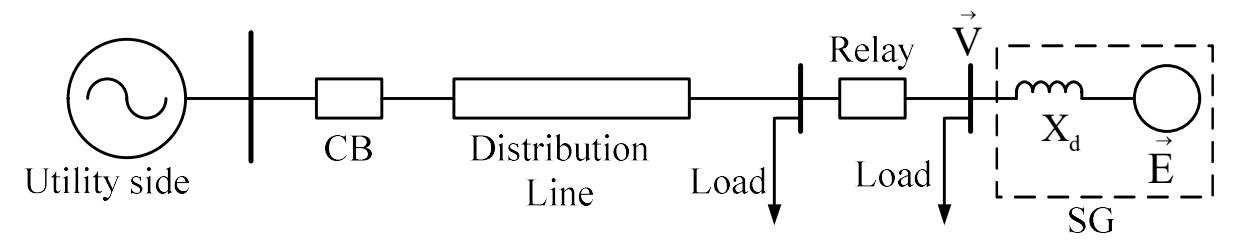

Fig. 1. A 3-bus radial distribution network with synchronous generator (SG) type DG.

From Fig. 2, it is noticeable that islanding provokes the variations of voltage magnitude and phase angle. Moreover, a change in frequency is also observed from the change of period of voltage cycle. Therefore, in the proposed method, five variables are employed for feature extraction: frequency $(f)$, rate of change of frequency $\left(\rho_{f}=d f / d t\right)$, rotor angle $(\delta)$, voltage $(V)$ in pu, and rate of change of voltage $\left(\rho_{v}=d V / d t\right)$. Five features are extracted from these five variables, by taking Standard Deviation (SD) inside a sliding data-window having a width of $\Delta T$. For instance, feature from a signal $s(t)$, which can be any of the five network variables, are extracted by taking SD inside the $\Delta T$ width of a sliding data-window. Following this procedure, five features are extracted from five network variables, which are obtained during islanding and non-islanding situations such as capacitor switching, load switching etc., see Fig. 3 for illustration.

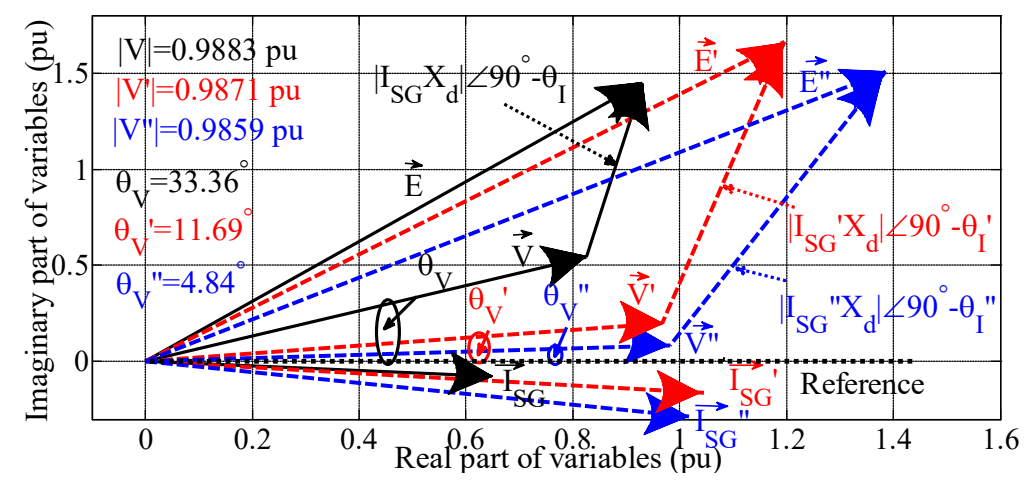

Fig. 2. Phasor diagram representing the voltage behavior at DG connection point of the system shown in Fig. 1 during pre- and post-islanding condition.

Mathematically, the five features can be presented as follows:

$$
\sigma_{v}=\operatorname{std}\{v(\tau) ; \tau \in[t-\Delta T, t]\}
$$




$$
\begin{aligned}
& \sigma_{f}=\operatorname{std}\{f(\tau) ; \tau \in[t-\Delta T, t]\} \\
& \sigma_{\delta}=\operatorname{std}\{\delta(\tau) ; \tau \in[t-\Delta T, t]\} \\
& \sigma_{\rho_{v}}=\operatorname{std}\left\{\frac{d v(\tau)}{d t} ; \tau \in[t-\Delta T, t]\right\} \\
& \sigma_{\rho_{f}}=\operatorname{std}\left\{\frac{d f(\tau)}{d t} ; \tau \in[t-\Delta T, t]\right\}
\end{aligned}
$$

where $\sigma_{v}, \sigma_{f}, \sigma_{\delta}, \sigma_{\rho v}$ and $\sigma_{\rho f}$ represent the features extracted from the network variables: voltage $(V)$, frequency $(f)$, rotor-angle $(\delta)$, rate-of-change-of-voltage $\left(\rho_{v}\right)$ and rate-of-change-of-frequency $\left(\rho_{f}\right)$, respectively. Therefore, the feature vector is given by

$$
\boldsymbol{x}=\left[\begin{array}{lllll}
\sigma_{v} & \sigma_{f} & \sigma_{\delta} & \sigma_{\rho_{v}} & \sigma_{\rho_{f}}
\end{array}\right]^{T}
$$

where $[.]^{T}$ denotes the transpose operator.
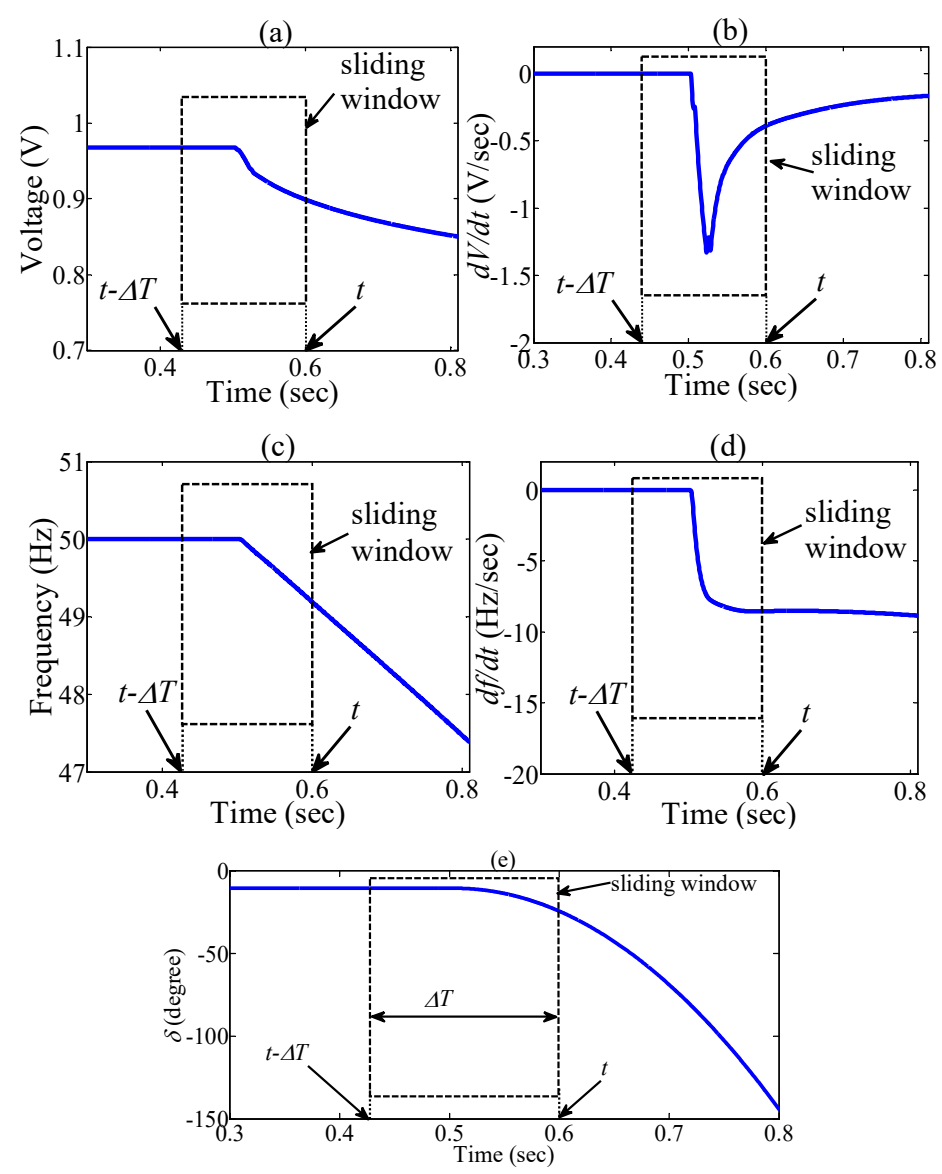

Fig. 3. Illustration of feature extraction from five network variables: (a) $V$, (b) $d V / d t$, (c) $f$, (d) $d f / d t$, (e) $\delta$; using a sliding data-window of $\Delta T$ width. 


\subsection{The Support Vector Machine Classifier}

In the present analysis, two groups of data, extracted from islanding and non-islanding events, are classified using support vector machines (SVMs). Therefore, a brief overview of the theory of SVM, proposed by Vapnik and co-workers [14]-[15], is presented in this subsection.

For a two-class classification problem, a real valued input or feature vector $\left(\boldsymbol{x}_{n} \in \Re^{d}\right)$ can be labeled as $y_{n}=\{-1,+1\}$, which indicates the class of $\boldsymbol{x}_{n}$. The SVM separates the two classes by establishing a decision boundary hyperplane defined by its normal vector $\boldsymbol{w}$ and a scalar bias $b$,

$$
\mathrm{g}(\boldsymbol{x})=\boldsymbol{w}^{\mathrm{T}} \boldsymbol{x}+b
$$

The function $\operatorname{sgn}\left(\boldsymbol{w}^{\mathrm{T}} \boldsymbol{x}+b\right)$ can be used as a decision function to obtain an output $y_{n}=-1$ or +1 , indicating the class of the input feature vector $\boldsymbol{x}_{n}$. The optimal separating hyperplane is the one with minimum Euclidean norm of $\boldsymbol{w}$, satisfying $y_{n}\left(\boldsymbol{w}^{T} \boldsymbol{x}_{n}+b\right) \geq 1$. Training of the SVM amounts to solving the following quadratic program $(\mathrm{QP})$ :

$$
\begin{array}{cll}
\underset{w, \xi}{\operatorname{minimize}} & \frac{1}{2}\|\boldsymbol{w}\|^{2}+C \sum_{n=1}^{N} \xi_{n} \\
\text { subject to } & y_{n}\left(\boldsymbol{w}^{T} \boldsymbol{x}_{n}+b\right) \geq 1-\xi_{n} & \text { for } n=1,2, \ldots . N \\
& \xi_{n} \geq 0 & \text { for } n=1,2, \ldots . N
\end{array}
$$

where $\xi_{n}$ is a slack variable and the scalar $C$ is a regularization parameter, which determines the trade-off between the maximization of the margin and the minimization of classification errors.To solve the QP problem of (8), Lagrange multipliers $\beta_{n} \geq 0$ and $\gamma_{n} \geq 0$ are introduced, which yields $\boldsymbol{w}$ in the form:

$$
\boldsymbol{w}=\sum_{n=1}^{N} \beta_{n} y_{n} \boldsymbol{x}_{n}
$$

Clearly, $\boldsymbol{w}$ is determined by the training data corresponding to Lagrange multipliers $\beta_{n}$ which are non-zero and for which the constraints in (8) are exactly met; these training samples are known as support vectors (SVs). The final decision boundary $g(\boldsymbol{x})$ can be expressed as,

$$
g(\boldsymbol{x})=\sum_{\beta_{n}>0} \beta_{n} y_{n} \boldsymbol{x}_{n}^{T} \boldsymbol{x}+b
$$

where $\boldsymbol{x}$ is the input test vector. As indicated earlier, the decision function can be taken as $\operatorname{sgn}(\mathrm{g}(\boldsymbol{x}))$, with a tuning parameter $C$. 
The kernel trick is also incorporated with SVMs to classify nonlinearly separable classes. Therefore, replacing the inner product $\boldsymbol{x}_{n}^{T} \boldsymbol{x}$ in (10) with the kernel function $K\left(\boldsymbol{x}_{n}, \boldsymbol{x}\right)$ yields the decision function:

$$
g(\boldsymbol{x})=\sum_{\text {SVs or } \beta_{n}>0} \beta_{n} y_{n} K\left(\boldsymbol{x}_{n}, \boldsymbol{x}\right)+b
$$

Some popular choices of kernel functions used with SVMs include

- Radial basis function (RBF) kernel: $K(x, y)=\exp \left\{-\frac{|x-y|^{2}}{2 \sigma^{2}}\right\}$

- Polynomial kernel of degree $p: K(x, y)=\left(x^{T} y+1\right)^{p}$

The kernel parameters $p$ and $\sigma$, along with $C$, are used as input parameters to the SVM training process; they are tuned to achieve the desired trade-off between training and generalization performance.

\subsection{Training and Testing of SVM for Islanding Detection}

Training of SVM is conducted off-line by extracting the input features from all possible scenarios of islanding and non-islanding events which may occur in DG networks. For each non-islanding and islanding events, the feature vector $\boldsymbol{x}$, as presented in Section 2.1, is obtained from a window of width $\Delta T$. In the training phase, the location of the onset of islanding is known a priori (ground truth); therefore, any window that includes the islanding onset is considered as islanding case. Thus, two groups of labelled data: islanding and non-islanding, are stored in a feature matrix. Then, soft-margin support vector classification algorithm employing Quadratic Programming (QP) optimization is applied. Cross-validation is conducted on the training set to obtain the optimum values of the regularization parameter $C$, kernel parameters $\sigma$ (for Gaussian RBF kernel) and order of polynomial $p$ (for polynomial kernel).

Testing of SVM is carried out by using the features extracted from a different set of islanding and non-islanding events, generated separately from the training set. Performance of the SVM classifier is evaluated using the Detection Rate (DR) and False Alarm (FA), where DR indicates the ratio of the number of successfully detected islanding events to the total number of islanding events, and FA indicates the ratio of the number of misclassified non-islanding events to the total number of non-islanding events.

\section{Test Results of the Proposed Approach}

The proposed machine learning approach is assessed for the islanding detection of a test network energized with synchronous type DG units at distribution feeder. The detailed description of the test system, generation of plausible islanding and non-islanding test cases or events, and their classification results are presented in the following three sub-sections. 


\subsection{Test System}

The SVM based approach is tested by the events generated through the simulation of a test network of Fig. 4. MATLAB/SIMPOWER software is used to build the test system model. The sampling frequency during the simulation study is kept at $2 \mathrm{kHz}$, and the relaysof circuit breakers are placed at the connection points of transformers adjacent to $\mathrm{SG}_{1}, \mathrm{SG}_{2}$ and $\mathrm{SG}_{3}$. These relays are used to collect the voltage signal during islanding and non-islanding conditions. Note that the simulation study involves the sampling rate of $2 \mathrm{KHz}$, therefore, the relays at Circuit Breaker's end would receive the voltage at $2 \mathrm{KHz}$ sampling rate (i.e. 40 samples/cycle for $50 \mathrm{~Hz}$ system); this sampling rate is realistic in power system, since for phasor measurement unit (PMU), which is a reliable device for measuring the voltage in electricity grids, can process 10 to 256 samples in each cycle for $50 \mathrm{~Hz}$ system.

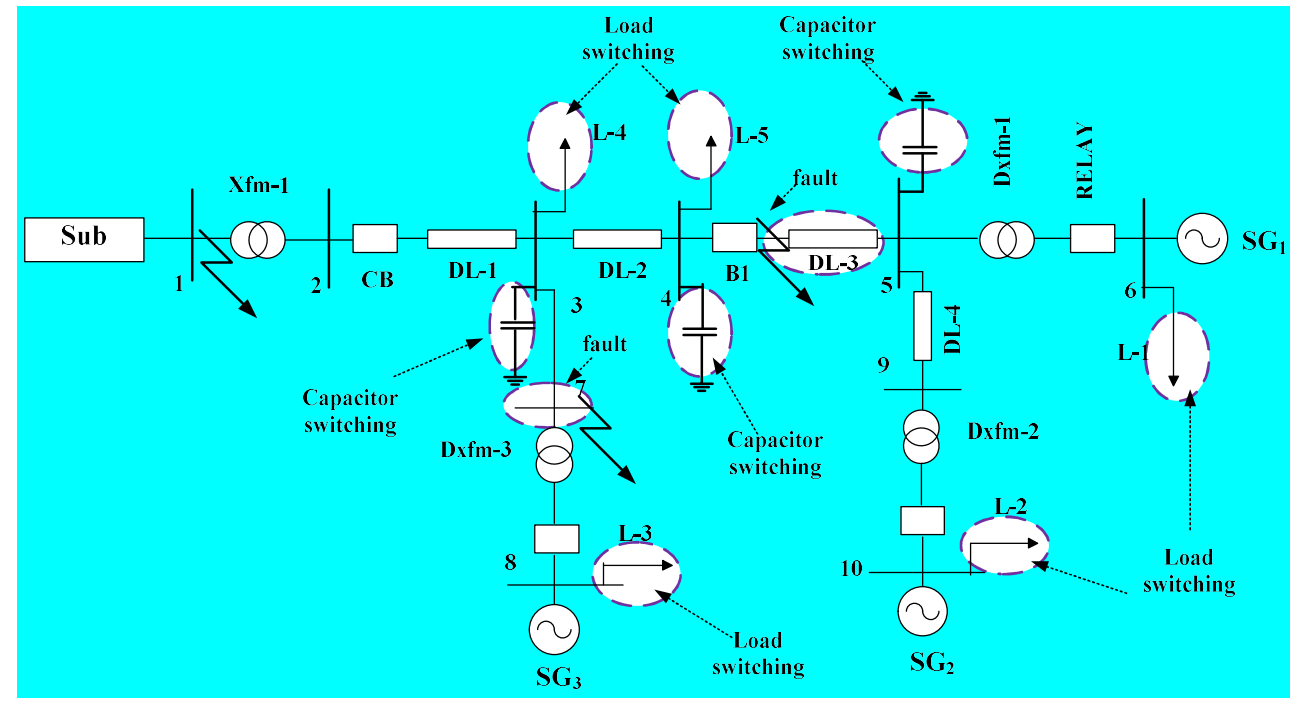

Fig.4. Single line diagram of a 10-bus radial distribution network under study.

As illustrated in Fig. 4, the test system is a radial distribution network having base power of 18 MVA. The distribution network is connected with $132 \mathrm{kV}, 50 \mathrm{~Hz}$, subtransmission system with fault level of 1000 MVA, shown by a Thévenin equivalent (Sub). A 33-kV distribution system is connected with the sub-transmission system or grid side through a 132/33-kV transformer. There are three 6-MW, 1.2-MVAr synchronous generators (SGs) connected to the distribution system through 33/0.69 kV transformers at bus 6, 8 and 10 respectively (see Fig. 4). For this simulation study, three-phase models of all network components are used. The $\pi$ section lines are modeled as distribution lines. Loads are modeled as dynamic loads which are of constant impedance, constant current and constant power type. The synchronous generator is represented by a sixth-order three-phase model in the $d q$ rotor reference frame [16] and it is 
equipped with an AVR (Automatic Voltage Regulator) represented by the IEEE-Type 1 model. More information about the test system can be obtained from [5].

\subsection{Test Cases/Events}

The network events that result in the isolation of the DG energized distribution network from the supply of the upstream network (or grid side) are considered as islanding conditions. Normal events that may persist in real power systems due to normal operation or disturbances, such as, capacitor switching, loss of lines, load addition, load disconnection, faults, etc., for which DG energized network is not isolated, are considered as non-islanding conditions. In this study, simulation of non-islanding cases include: 1) normal operation or normal condition, 2) temporary faults which include balanced three-phase faults, unbalanced single and double-phase faults, 3) switching of capacitor banks, 4) switching due to addition and/or disconnection of loads, 5) Disconnection of DGs apart from the target DG, 6) Loss of any branch in the radial distribution feeder, which is away from the distribution line connected to the target DG. The islanding cases are simulated by opening the circuit breaker (CB) or feeder breaker (B1) of Fig. 4 under the following network conditions [17]:

1) Containing wide range of active power imbalance (deficit and excess, varying from $0 \%$ to $100 \%$ ) in the islanded portion,

2) Containing wide range of active power imbalance (deficit and excess, varying from $0 \%$ to $100 \%$ ) and reactive power imbalance (deficit and excess, varying from $0 \%$ to $50 \%$ ) in the islanded portion, and

3) Containing three types of loads: constant impedance, constant current and constant power.

In order to generate the islanding events with a wide range of active and reactive power imbalance, the load-generation profile in the islanded segment is varied by applying the procedure presented in [17].

\subsection{Classification of Events using SVM}

A total of 2,817 events (see Table 1) are generated for assessing the classification performance of SVM. It is worth noting that the generated islanding events have covered all four probable combinations or groupings of power imbalance scenarios; which include, (a): Deficit of $\Delta \mathrm{P}$ and Deficit of $\Delta \mathrm{Q}$, (b): Deficit of $\Delta \mathrm{P}$ and Excess of $\Delta \mathrm{Q}$, (c): Excess of $\Delta \mathrm{P}$ and Excess of $\Delta \mathrm{Q},(\mathrm{d})$ : Excess of $\Delta \mathrm{P}$ and Deficit of $\Delta \mathrm{Q}$ [9]. Table 1show the numbers of training and test events, which reveal that the number of generated islanding events is greater than the number of non-islanding events. Note that the probability of occurring islanding incidents is rare in the distribution network in comparison to the non-islanding incidents. However, in this 
work, the probability of islanding occurrence is not considered; rather the different scenarios of islanding and non-islanding cases are taken into account. Furthermore, in comparison to testing data less number of training data is used. As stated earlier, the training data is selected as a different subset from the test data. For the generation of islanding training data, all possible combinations of power imbalance scenarios, where $\Delta \mathrm{P}$ ranges from $0 \%$ to $100 \%$ (step size $4.8 \%$ ) and $\Delta \mathrm{Q}$ ranges from $0 \%$ to $50 \%$ (step size $10 \%$ ), are considered. Thus, a total of 249 islanding events are generated for training. The training data is used to obtain the optimum parameters of the SVM classifier; see Section 2.3 for further illustration. In order to investigate the effectiveness of the proposed method, the trained SVM is tested on a large number of separate islanding and non-islanding events (consisting of 1,848 islanding and 471 non-islanding): the test events are generated by varying $\Delta \mathrm{P}$ from $0 \%$ to $100 \%$ (step size $0.5 \%$ ) and $\Delta \mathrm{Q}$ from $0 \%$ to $50 \%$ (step size $10 \%)$.

The performance of SVM classifier is assessed from the classification results of numerous events of Table 1, by using the five features extracted through a window of $\Delta \mathrm{T}$ width (see section 2.1 ). The width of the window is optimally selected by conducting the SVM based classification, using the features extracted from the situations having window-width $(\Delta \mathrm{T})$ of one-cycle, five-cycle, eight-cycle and ten-cycle. For each situation, the threshold value of SVM classifier is varied gradually to obtain the Detection Rate (DR) and False Alarm (FA). Thus, the Receiver Operating Characteristics (ROC) curve, as shown in Fig. 5, is obtained.

Table 1 Generated islanding and non-islanding events under different combinations of power imbalance level

\begin{tabular}{|c|c|c|c|c|}
\hline \multirow{2}{*}{ Scenarios } & \multicolumn{2}{|c|}{ Islanding events } & \multicolumn{2}{|c|}{ Non-islanding events } \\
\hline & Training & Test & Training & Test \\
\hline (a): Deficit $\Delta \mathrm{P}$ and Deficit $\Delta \mathrm{Q}$ & 63 & 462 & \multirow{4}{*}{249} & \multirow{4}{*}{471} \\
\hline (b): Deficit $\Delta \mathrm{P}$ and Excess $\Delta \mathrm{Q}$ & 62 & 462 & & \\
\hline (c): Excess $\Delta \mathrm{P}$ and Excess $\Delta \mathrm{Q}$ & 62 & 462 & & \\
\hline (d): Excess $\Delta \mathrm{P}$ and Deficit $\Delta \mathrm{Q}$ & 62 & 462 & & \\
\hline Total & 249 & 1848 & 249 & 471 \\
\hline
\end{tabular}

The ROC curve of Fig. 5 indicates that the classifier's performance using eight-cycle and ten-cycle data-window are almost similar; and their performances are comparatively better than the five-cycle and one-cycle data-window for FA $\leq 5 \%$. However, considering the response time and performance, eightcycle data-window is selected as optimal data-window to investigate the performance of the proposed method. Thus, using the extracted features through eight-cycle data-window and applying the SVM classifier with linear, polynomial and Gaussian RBF kernels, the test islanding and non-islanding events are classified. The bound on the regularization parameter " $C$ " is selected as 10 after performing the grid- 
search using the SVM classifier. The test results presented in Table 2 show that linear and polynomial kernel yield almost similar performance for scenarios (a) and (b), considering DR and FA. However, taking all four power imbalance scenarios into account, polynomial kernel shows the best classification performance among the three kernels, as indicated in Table 2.

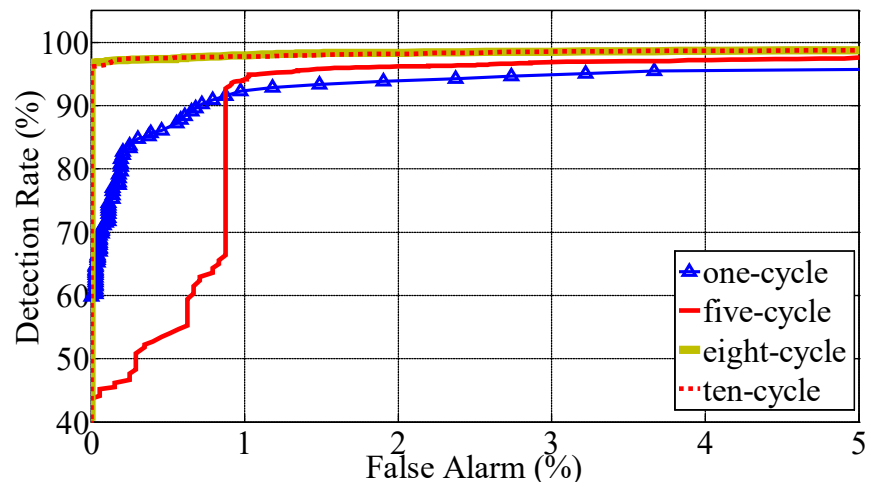

Fig. 5. ROC curve of the proposed approach for one-cycle, five-cycle, eight-cycle and ten-cycle data-window length.

Table 2 Performance of SVM classifier including all four combinations of deficit of active/reactive and excess of active/reactive power imbalance

\begin{tabular}{|c|c|c|c|c|c|c|c|c|c|}
\hline \multirow{2}{*}{ Kernels } & \multirow{2}{*}{$\begin{array}{c}\text { Kernel pa- } \\
\text { rameter }\end{array}$} & \multicolumn{2}{|c|}{ Scenario (a) } & \multicolumn{2}{c|}{ Scenario (b) } & \multicolumn{2}{c|}{ Scenario (c) } & \multicolumn{2}{c|}{ Scenario (d) } \\
\cline { 3 - 10 } & & DR (\%) & FA (\%) & $\begin{array}{c}\text { DR } \\
(\%)\end{array}$ & FA (\%) & DR (\%) & FA (\%) & DR (\%) & FA (\%) \\
\hline Linear & - & 100 & 3.08 & 100 & 3.08 & 94.7 & 3.08 & 94.1 & 3.08 \\
\hline Gaussian RBF & $\sigma=1.5$ & 94.6 & 3.34 & 93 & 3.34 & 98.2 & 3.34 & 98.8 & 3.34 \\
\hline Polynomial & $p=3$ & 100 & 3.29 & 100 & 3.29 & 99.2 & 3.29 & 99.7 & 3.29 \\
\hline
\end{tabular}

\section{Performance Evaluation and Comparative Analysis}

Islanding detection tool has to be implemented in real-time application. Therefore, speed and detection-time of the SVM based algorithm embedded relay (SVMR) needs to be investigated. In this context, speed implies the processing speed of SVMR and it is expected to be fast, given the fact that the real-time extracted features are passed through the trained SVM containing a small number of support vectors. Detection-time of relay is defined as the time-delay, which starts soon after the onset of islanding and finishes as soon as islanding is detected. In the following sub-sections, firstly, the performance of the proposed approach is evaluated considering the reliability, which comprises the detection rate (DR), false alarm (FA), and the response time or detection-time of islanding. Then, SVM based machine learning approach is compared with conventional relays under all possible power imbalance scenarios that could be present during islanding. Finally, a general discussion is presented in Section 4.3. 


\subsection{Performance Assessment of the Proposed Machine Learning Approach}

It should be noted that the test results of Table 2 were achieved with the features extracted from a fixed window width of 8-cycle, since 8-cycle data-window was selected as an optimum trade-off window considering response time, DR and FA as illustrated in Fig. 5. In this investigation, the event (islanding/non-islanding) inception time was known a priori; therefore, the starting point of the window was considered from the event inception-time and the end point of the window was 8 cycles after the event onset. However, in practice, the event (islanding/non-islanding) has to be detected in real-time without prior knowledge of inception-time. Therefore, to examine the performance of SVM more critically, an 8cycle long sliding window is considered with a step-size of one-cycle and classification results are recorded, see Fig. 6 for illustration. For example, if an 8-cycle window includes 1-cycle data after the onset of islanding, then DR is calculated; likewise, if the window includes 1-cycle posterior non-islanding data then FA is estimated. Following a similar approach, using 8-cycle data-window containing 2-cycle, 3cycle, 4-cycle, 5-cycle, 6-cycle, 7-cycle, and 8-cycle of posterior events, DR and FA are calculated and the results are illustrated in Fig. 7. Figs. 7(a)-7(d) demonstrate the FA and DR for four possible power imbalance scenarios when data-window includes 1-cycle to 8-cycle posterior events (islanding/nonislanding). Moreover, in order to assess the classification performance in more details, F-measure is calculated and presentedin Fig. 7. Note that F-measure is an indicator for the classifier's performance; it is given byF-measure $=2 \mathrm{TP} /(2 \mathrm{TP}+\mathrm{FP}+\mathrm{FN})$, where $\mathrm{TP}=$ True Positive, $\mathrm{FP}=$ False Positive, $\mathrm{FN}=$ False Negative and TN =True Negative [18]. In this work, TP indicates the successful classification of islanding events, FP implies the misclassification of non-islanding events, TN indicates the successful classification of non-islanding events and FN specifies the misclassification of islanding events.

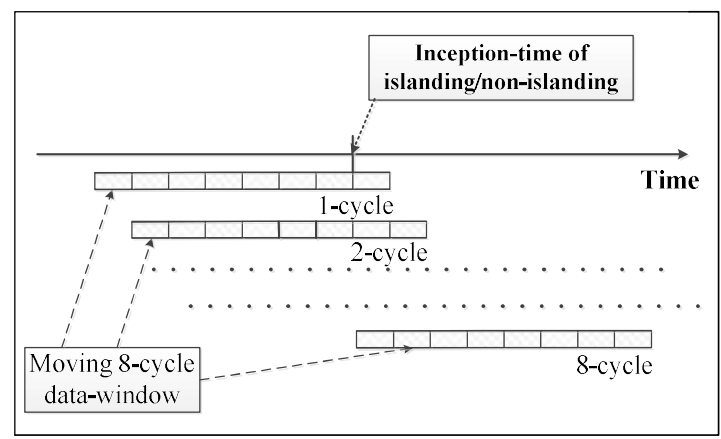

Fig. 6. Data-stream for illustrating the concept of moving 8-cycle data window and its classification at each step (1-cycle to 8cycle). 
(a)

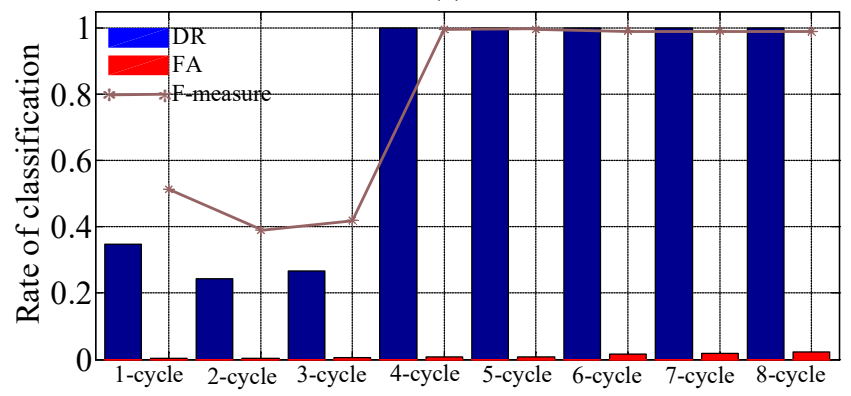

(c)

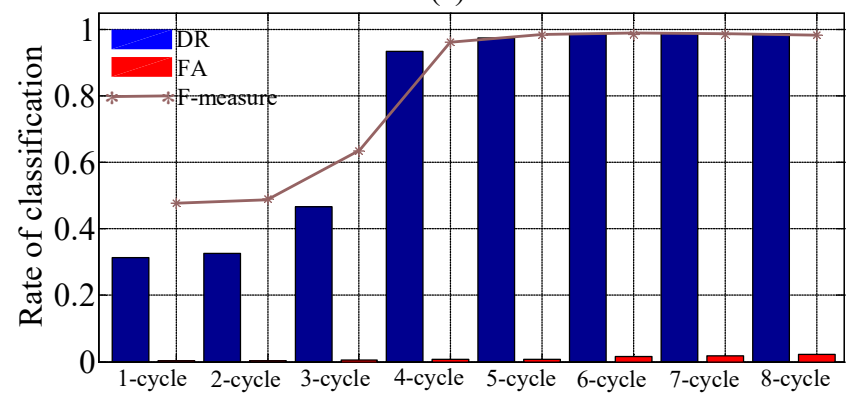

(b)

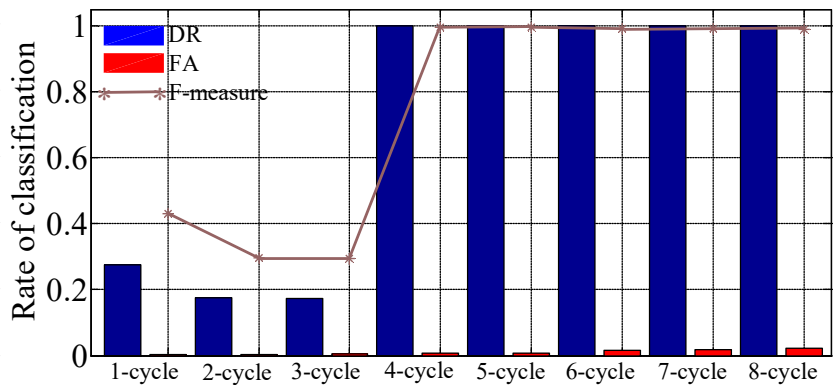

(d)

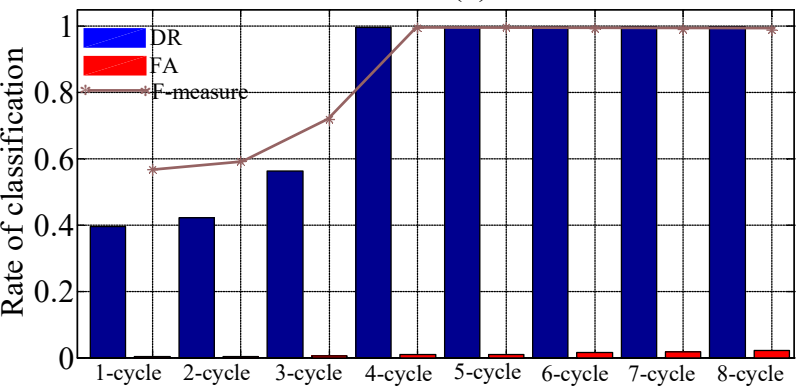

Fig. 7. Classification results(F-measure, DR, FA) using 8-cycle moving data-window including data-stream of 1 cycle to 8cycle posterior events under scenarios (a) Deficit $\triangle P$ and deficit $\triangle Q$, (b) Deficit of $\triangle P$ and excess of $\triangle Q$, (c) Excess of $\triangle P$ and excess of $\triangle Q$, and (d) Excess of $\triangle P$ and deficit of $\Delta Q$.

From the results of Figs. 7(a)-7(d), it is observed that when 8-cyle data-window includes 5-cycle data of posterior events (islanding/non-islanding), then F-measure and its associated DR and FA show comparatively better performance under all four power imbalance scenarios. Hence, in order to summarize the classification results under all possible scenarios, F-measure, DR, FA, FN and TN are shown in Fig. 8.

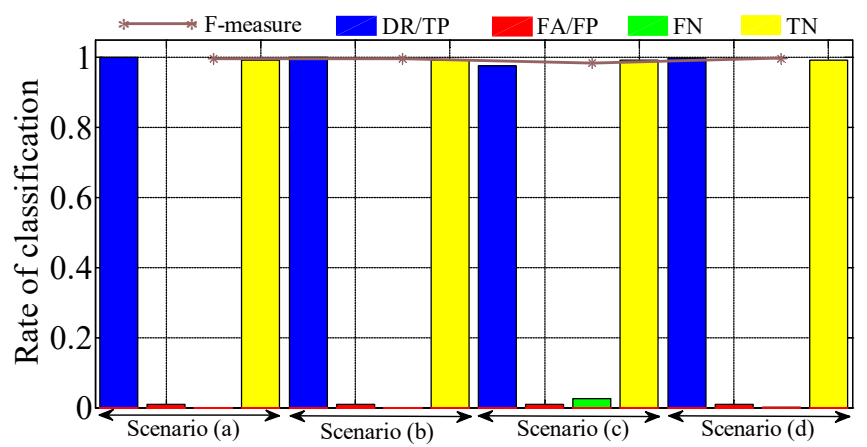

Fig. 8. Classification results using 8-cycle data-window including data-stream of 5 cycle posterior events under scenarios (a) Deficit $\triangle P$ and $\triangle Q$, (b) Deficit of $\triangle P$ and excess of $\triangle Q$, (c) Excess of $\triangle P$ and $\triangle Q$, and (d) Excess of $\triangle P$ and deficit of $\Delta Q$.

From Fig. 8 it is evident that $100 \%$ DR with $0 \%$ FA is not achieved for all possible power imbalance scenarios. However, for practical application, if $100 \%$ DR and $0 \%$ FA is required, then a majority voting 
rule along with the SVM is proposed. The proposed rule guarantees $100 \%$ DR and $0 \%$ FA with a minor relaxation in the response or detection time; the details are discussedbelow.

In order to investigate the decision-rule based on majority voting, sliding data-window of eight-cycle width is moved by a typical step-size of one cycle, and the features are extracted inside each window block or sample to classify the event as islanding or non-islanding. To accomplish this task, firstly, features of the generated islanding and non-islanding events are considered with known event inception-time (groundtruth). Then eight-cycle data-window, which starts from 15 cycles prior to event inception, are moved by one cycle step-size until 20 cycles posterior of event inception-time is reached. Thus, 28 samples are obtained for each event including islanding and non-islanding. These samples are classified using the trained SVM and decisions are made based on the majority voting of the classification results of consecutive samples, for example, 3, 5, 7 or 9 samples. Moreover, the decision-rule based on the classification results of 1 sample, which does not require majority voting, is considered. Higher number of samples, e.g., 11 or higher, may also be investigated for the decision making process; however, it would require higher detection-time and, eventually makes it less practical option. It should be noted that odd number of samples are considered for the decision-rule, since it simplifies the decision making process; for example, 9 samples decision-rule requires at least 5 samples to be classified as islanding for the detection of an actual islanding event. Again, note that majority voting of 9 samples consider the previous consecutive 9 samples in descending order; and it implies that in time-domain, the rightmost end of eightcycle data-window of $9^{\text {th }}$ sample specifies the instant of "event classification time" or detection-time. Decision-rule of 3, 5 and 7 samples follow the same technique as used for 9 samples' decision-rule.

Fig. 9 illustrates the DR and FA of the proposed approach, using sliding data-window of eight-cycle width and applying the decision rule based on the majority voting of the classification results of 9 samples, 7 samples, 5 samples, 3 samples and one sample. In Figs. 9(a)-9(b), the $x$-axis is the "event classification time" which starts soon after the inception of islanding and non-islanding events, respectively. From Fig. 9(a) it is evident that one sample decision rule can yield almost $100 \%$ DR within $100 \mathrm{~ms}$ of islanding inception. But, this decision rule imposes the risk of 1\% FA after $100 \mathrm{~ms}$ of event (non-islanding) inception; and it can reach to a maximum of 7\% FA after $250 \mathrm{~ms}$ (see Fig. 9(b)). The decision rule, obtained from the majority voting of 5 consecutive samples, gives the performance level in between 9 samples and one sample. In summary, considering the reliability and keeping a trade-off between response-time and the classification results (i.e., DR and FA), 9 samples decision rule yields comparatively better performance than the decision rule of 7, 5, 3 samples and one sample, as highlighted in Figs. 9(a)-9(b). 
(a)
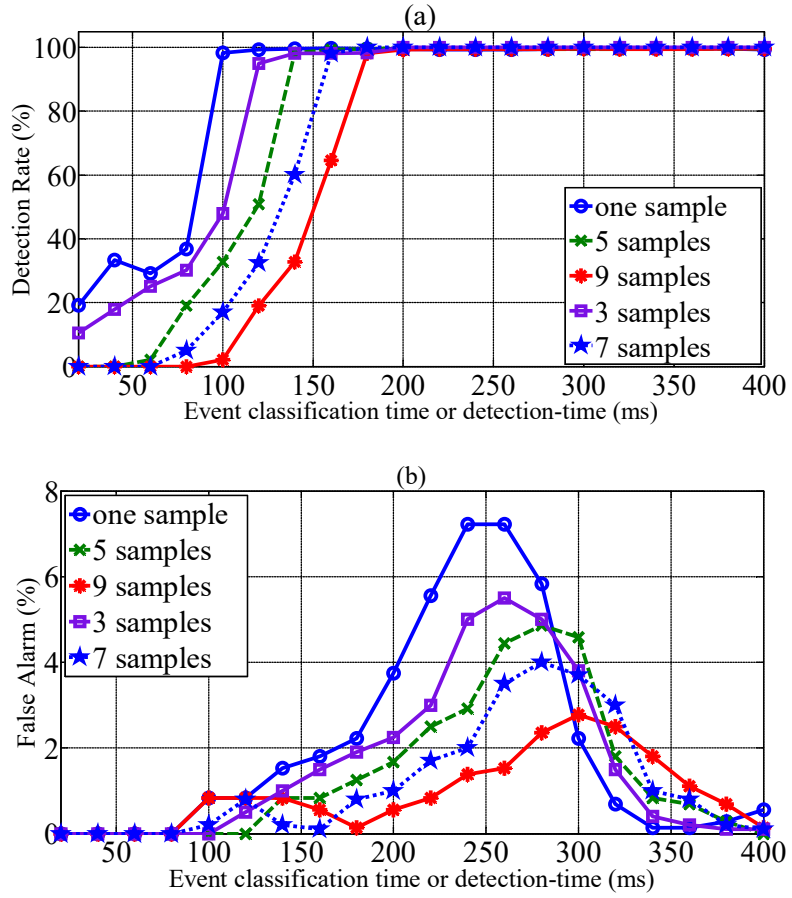

Fig. 9. Performance of the proposed SVM based approach, indicated by (a) Detection Rate and (b) False Alarm, using decision-rule of 9 samples, 7 samples, 5 samples, 3 samples and one sample.

Table 3 Islanding detection time of proposed SVM based method employing 9-samle majority voting rule under all possible power imbalance scenarios

\begin{tabular}{|c|c|c|c|}
\hline Scenarios & $\begin{array}{c}\text { Maximum detection } \\
\text { time }\end{array}$ & $\begin{array}{c}\text { Minimum detection } \\
\text { time }\end{array}$ & $\begin{array}{c}\text { Average detection } \\
\text { time }\end{array}$ \\
\hline Scenario (a): Deficit of $\Delta \mathrm{P}$ and deficit of $\Delta \mathrm{Q}$ & $180 \mathrm{~ms}$ & $120 \mathrm{~ms}$ & $153 \mathrm{~ms}$ \\
\hline Scenario (b): Deficit of $\Delta \mathrm{P}$ and excess of $\Delta \mathrm{Q}$ & $180 \mathrm{~ms}$ & $100 \mathrm{~ms}$ & $155 \mathrm{~ms}$ \\
\hline Scenario (c): Excess of $\Delta \mathrm{P}$ and excess of $\Delta \mathrm{Q}$ & $300 \mathrm{~ms}$ & $120 \mathrm{~ms}$ & $161 \mathrm{~ms}$ \\
\hline Scenario (d): Excess of $\Delta \mathrm{P}$ and deficit of $\Delta \mathrm{Q}$ & $180 \mathrm{~ms}$ & $100 \mathrm{~ms}$ & $150 \mathrm{~ms}$ \\
\hline
\end{tabular}

From Fig. 9 it is observed that 9 -samples majority rule can guarantee $100 \%$ DR with approximately $0 \%$ FA, but not exactly 0\% FA. Moreover, for the investigation shown in Fig. 9, a step-size of one cycle has been considered for the sliding window, see Fig. 6 for illustration. The step-size is a configurable parameter, which can be varied. Investigating 0.5 cycle, 1 cycle, 1.5 cycle, and 2 cycle of step-size for the sliding data-window along with 9 -samples majority voting rule, it is found that $100 \%$ DR with exactly $0 \%$ FA is achieved with a step-size of 1.5-cycle. Therefore, employing 9-samples majority voting rule along with step-size of 1.5 -cycle, $100 \%$ DR and $0 \%$ FA is achieved. Table 3 shows the maximum, minimum and average response time for islanding detection under all four power imbalance scenarios. Different combination of power imbalance may exist during islanding events and considering four possible scenarios, all islanding events were successfully detected employing 9-sample majority voting rule. 
However, the detection or response time varies differently for different scenarios where the minimum and maximum detection time lies within $100 \mathrm{~ms}$ to $300 \mathrm{~ms}$. Note that the maximum detection time of $300 \mathrm{msis}$ below the IEEE standard permissible time of 2 seconds.

It is worth noting that power imbalance level has a relationship with the detection time and Table 3 does not show the variation of detection time as a function of power imbalance level. Hence, Section 4.2 demonstrates the detection/response time under all possible scenarios as a function of power imbalance level using the 9 samples decision rule with the proposed SVM method. In addition, performance of vector surge, ROCOF and frequency relays are presented in Section 4.2 for comparison with with the proposed algorithm.

\subsection{Comparative Analysis of the Proposed Method with Conventional Relays}

Conventional relays, such as, under/over frequency relay (FR), vector surge relay (VSR), rate-ofchange-of-frequency (ROCOF) relay, can be recognized as representative examples of islanding detection devices [9]. In [1], the frequency tripping requirements are recommended for distributed generators under abnormal frequencies; a standard DG protection scheme must satisfy the frequency tripping requirements as well as the anti-islanding requirements simultaneously. Thus, time-delay settings of $100 \mathrm{~ms}$ can be used [19]. FR measures the cycle-duration of the terminal voltage by using voltage zero-crossing detection technique and signal processing method. For ROCOF relay, rate-of-change-of-frequency is calculated considering a measured window over a few cycles, usually between 2 and 50 cycles. VSR relays measure the duration of an electrical cycle of terminal voltage and compare the current cycle duration with the last one. If this comparison gives the variation of cycle duration to be greater than a pre-specified threshold, then VSR relay is tripped [9]. Note that these relays or protection devices are disabled if the magnitude of the terminal voltage falls below a specific threshold to avoid false operation. As demonstrated in [9], settings of VSR and ROCOF relays with very high sensitive values, e.g., settings of $2^{\circ}-5^{\circ}$ for VSR relays and $0.1-0.25 \mathrm{~Hz} / \mathrm{s}$ for ROCOF relays, may create nuisance tripping. Therefore, relay setting of $10^{\circ}$ for VSR, $0.5 \mathrm{~Hz} / \mathrm{s}$ for ROCOF, and deviation of $\pm 0.5 \mathrm{~Hz}$ from nominal frequency for under/over frequency relays [20], are considered for the comparative analysis of these conventional protection devices with the SVM based relay (SVMR).

Figs. 10(a)-10(d) illustrate the islanding detection time of SVM relay (SVMR), frequency relay (FR), vector surge relay (VSR) and ROCOF relay, during all probable combinations of power imbalance scenarios, which include deficit and excess of active power imbalance $(\Delta \mathrm{P})$ and reactive power imbalance $(\Delta \mathrm{Q})$ inside the islanded network. In Fig. 10, $y$-axis refers to "detection time" which is considered as the 
time period after the onset of islanding. Islanding events, which are used for comparative analysis, are generated by keeping $\Delta \mathrm{P}=0.5 \%, 1 \%, 1.5 \%, \ldots, 100 \%$ (deficit or excess) in combination with $\Delta \mathrm{Q}=10 \%$, $20 \%, \ldots, 50 \%$ (deficit or excess) inside the islanded segment, by varying the load-generation profile and AVR setting of exciter model of synchronous generator. Thus, the islanding events corresponding to four combinations of power imbalance scenarios are obtained. Then "detection time" for each event is determined using the conventional relays as well as SVMR. Note that decision rule of SVMR has employed 9 samples majority voting due to its reliability, as demonstrated in Section 4.1.
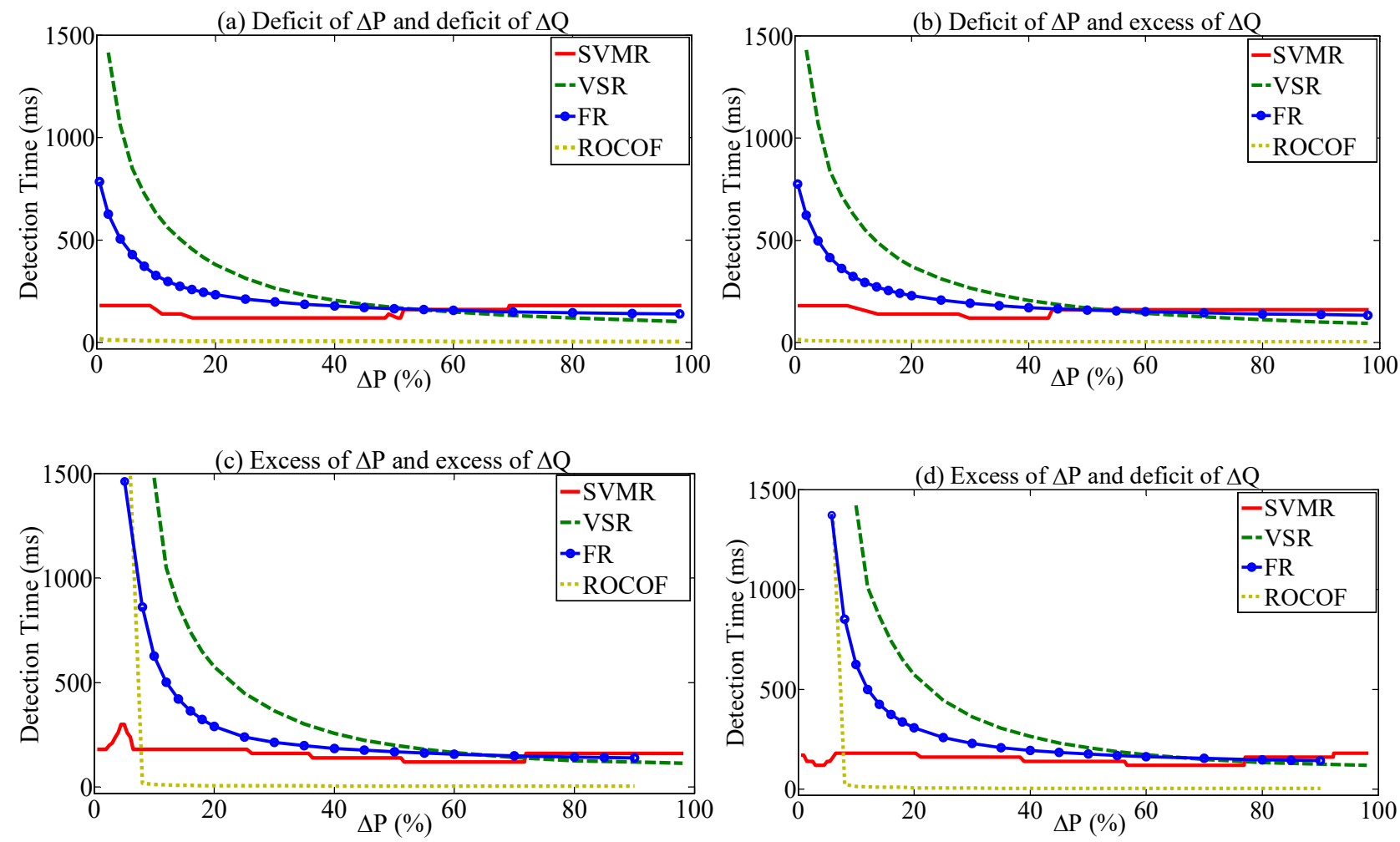

Fig. 10. Detection time of the proposed SVMR and other conventional relays - vector surge relay (VSR), frequency relay (FR) and ROCOF relay; under four possible combinations of power imbalance scenarios: (a), (b), (c) and (d).

From Figs. 10(a)-10(d), it is revealed that islanding detection time of SVMR falls within the range of $120 \mathrm{~ms}$ to $300 \mathrm{~ms}$, which is much lower than the IEEE 1547-2003 recommended standard tripping times of 2 seconds. Moreover, SVMR relay can successfully operate under all possible combinations of power imbalance scenarios within $200 \mathrm{~ms}$ of the onset of islanding, as shown in Figs. 10(a)-10(d). However, SVMR requires a slightly higher detection time, i.e., 200-300 ms under the scenario of excess of $\Delta \mathrm{P}$ and $\Delta \mathrm{Q}$ with $\Delta \mathrm{P}<5 \%$ (see Fig. 10(c)). In comparison to SVMR, conventional relays: FR and VSR need larger detection time whereas ROCOF relays need smaller detection time, when the islanded system possesses a 
deficit of $\Delta \mathrm{P}$ falling within the range of $\Delta \mathrm{P}<15 \%$ along with deficit or excess of $\Delta \mathrm{Q}$, as highlighted in Figs. 10(a) and 10(b). However, the scenarios, depicting excess or deficit of $\Delta \mathrm{Q}$ and excess of $\Delta \mathrm{P}$ which lies inside the range of $\Delta \mathrm{P}<10 \%$, have revealed that ROCOF relays along with FR and VSR require much higher detection time than SVMR (see Figs. 10(c)-10(d)). Thus, analyzing the performance of SVMR, considering the detection time and effectiveness under all possible combinations of power imbalance scenarios, it can be concluded that the proposed SVM based relay, developed using the machine learning algorithm, can be an important tool to serve the purpose of islanding detection of synchronous type DG units.

\subsection{Discussion}

The SVM based classification technique exploits multiple features extracted from network variables. During islanding situation, variation of network variables is mainly influenced by the active and/or reactive power imbalance in the islanded network. Based on the variation of these features, SVM based technique classifies the event as islanding or non-islanding. In this work, all probable combinations of power imbalance scenarios, which include deficit and/or excess of active and/or reactive power imbalances, were taken into consideration. Therefore, even though this article investigated the performance of SVM based approach using synchronous generator based DG, it is expected that for other types of DG, for example, DFIG, PV, etc., the proposed algorithm would perform effectively if it is designed considering all probable combinations of power imbalance scenarios. To this end, a DFIG based Wind Farm (6 MW) is connected in bus 8 of Fig. 4 instead of $\mathrm{SG}_{3}$, and the islanding events under different power imbalance scenarios are generated. Test results indicate that all islanding events (monitored from DFIG Wind Farm) are successfully detected within $200 \mathrm{~ms}$ of islanding inception. In addition, in the interconnection standards, the most difficult condition for islanding detection occurs when both active and reactive power imbalance exist under high Quality factor $(\mathrm{Q})$ of loads, e.g., $\mathrm{Q}=1$ or $\mathrm{Q}=3$. In the proposed method, all possible power imbalance conditions along with different types of loads were taken into account. Hence, in the presence of any range of quality factor of the loads, $Q=1$ or $Q=3$, the proposed approach is expected to exhibit successful classification.

In this article, detection rate and false alarm were used as key performance indicators for evaluating the performance of the proposed approach. Furthermore, the proposed method is evaluated using Fmeasures. Using the proposed method, an F-measure above 0.95 was obtained by considering all possible islanding and non-islanding scenarios. 


\section{Conclusion}

This paper presents a SVM based machine learning approach for islanding detection of synchronous type DG units. The proposed approach employs multiple features which are extracted from network variables, and then, these features are fed to a support vector machine (SVM) classifier to classify the event as islanding or non-islanding. The feature extraction incorporates a sliding window; and the width of the window is optimally selected from the Receiver Operating Characteristics (ROC) curve of the SVM classifier using different window-width. Supervised SVM classifier with linear, polynomial and Gaussian RBF kernels are used to train the selected features of numerous islanding and non-islanding events, which are generated by simulating a test network. With the training data, grid search is carried out to fine-tune the kernel parameters (i.e. $p$ and $\sigma$ ), and accuracy of the classifiers is tested. The proposed method is examined considering the islanding events under all probable combinations of deficit of active/reactive and excess of active/reactive power imbalance scenarios inside the islanded network. The performance of SVM based relay (SVMR), which can be developed from the proposed algorithm, is evaluated considering the suitability in real-time operation. The evaluation index includes the reliability, which comprises the detection rate (DR), false alarm (FA), and the response time represented by the detection-time of islanding. In summary, the contributions of the paper can be highlighted as:

- This article assesses the performance of multiple features based SVM method employing windowsize with variable length; thereafter, keeping a trade-off between response-time and reliability, an optimal window-width is selected by observation.

- In practical situation, the inception-time of islanding is not known in advance. Therefore, to investigate the performance of the SVM based approach, in this article, sliding data-window of eight-cycle width is moved by a typical step-size of one cycle, and the features are extracted inside each window block to classify the event as islanding or non-islanding. Thereafter, a decision making rule based on majority voting is obtained by analysing the performance on the basis of detection rate and false alarm.

- Comparative analysis of SVMR with conventional relays, such as, vector surge relays, ROCOF and frequency relays, are conducted; and it shows that SVMR performs quite well in comparison to conventional relays when reliability and detection-time of islanding are taken into account.

\section{References}

[1] IEEE Standards Coordinating Committee 21: 'IEEE standard forinterconnecting embedded resources withelectric power systems',IEEE Standard 1547, 2003. 
[2] IEEE Application Guide for IEEE Std. 1547: 'IEEE Standard for Interconnecting Distributed Resources with Electric Power Systems', IEEE Std. 1547.2-2008, 2009.

[3] Lasseter, H. G. Far, A. J. Rodolakis, and G. Joos: 'Synchronous distributed generation islanding protection using intelligent relays', IEEE Trans. Smart Grid, Dec. 2012, 3, (4), pp. 1695-1703.

[4]L. K. Kumpulainen, and K. T. Kauhaniemi: 'Analysis of the impact of distributed generation on automatic reclosing'Proc. IEEE PESPower Syst. Conf. Expo., 2004, pp. 603-608.

[5] M. R. Alam, K. Muttaqi, A. Bouzerdoum: 'An Approach for assessing the effectiveness of multiple features based SVM method for islanding detection of distributed generation', IEEE Transactions on Industry Applications, July-Aug. 2014, 50, (4), pp. 2844-2852.

[6] E. Kamyab, and J. Sadeh: 'Islanding detection method for photovoltaic distributed generation based onvoltage drifting', IET Gen., Trans. \& Distribution, Jun. 2013, 7 (6), pp. 584-592.

[7] H. H. Zeineldin, and S. Conti: 'Sandia frequency shift parameter selection for multi-inverter systems toeliminate non-detection zone', IET Renewable Power Generation, Mar. 2011, 5 (2), pp. 175-183.

[8] A. Khamis, H. Shareef, and A. Mohamed: 'Islanding detection and load shedding scheme for radialdistribution systems integrated with dispersed generations', IET Gen., Trans. \& Distribution, Nov. 2015,9 (15), pp. 22612275.

[9] W. Freitas, Wilsun Xu, C. M. Affonso, and Zhenyu Huang: 'Comparative analysis between ROCOF and vector surge relays for distributed generation applications', IEEE Trans. Power Del., Apr. 2005, 20, (21), pp. 13151324.

[10] N. W. A. Lidula, and A. D. Rajapakse:'A pattern recognition approach for detecting power islands using transient signals - part I: design and implementation', IEEE Trans. Power Del., Oct. 2010, 25, (4), pp. 3070-3077.

[11] H. Bitaraf, M. Sheikholeslamzadeh, A. M. Ranjbar, and B. Mozafari:'Neuro-fuzzy islanding detection in distributed generation'Proc. IEEE Conference on Innovative Smart Grid Technologies - Asia (ISGT Asia), Tianjin, 21-24 May 2012, pp. 1-5.

[12] M. R. Alam, K. M. Muttaqi, and A. Bouzerdoum: 'A Multifeature-Based Approach for Islanding Detection of DG in the Subcritical Region of Vector Surge Relays', IEEE Transactions on Power Delivery, Oct. 2014, 29, (5), pp. 2349-2358.

[13] K. El-Arroudi, G. Joos, I. Kamwa, and D. T. McGillis: 'Intelligent-based approach to islanding detection in distributed generation', IEEE Trans. Power Del., Apr. 2007, 22, (2), pp. 828-835.

[14] V. N. Vapnik:'Statistical Learning Theory',New York: John Wiley, 1998.

[15] N. Cristianini, and J. Shawe-Taylor: 'An Introduction to Support Vector Machines and Other Kernel-based Learning Methods', Cambridge, MA: Cambridge Univ. Press, 2000.

[16] P.Kundur:'Power System Stability and Control', New York: McGraw-Hill, 1994. 
[17] Jose C. M. Vieira, Walmir Freitas, Wilsun Xu, and Andre Morelato:'An investigation on the nondetection zones of synchronous distributed generation anti-islanding protection', IEEE Trans. Power Del., Apr. 2008, 23, (2), pp. 593-600.

[18] T. Fawcett: 'An introduction to ROC analysis', Pattern Recognition Letters, Jun 2006, 27, (8), pp. 861-874.

[19] J. C. M. Vieira, W. Freitas, W. Xu, and A. Morelato: 'performance of frequency relays for distributed generation protection', IEEE Trans. Power Del., Jul. 2006, 21, (3), pp. 1120-1127.

[20] Technical Report of NECA for Frequency operating standards determination, Sep. 2001. [Available online] http://www.neca.com.au/Files/RP_Final_Determination_Sep_2001.pdf 